\title{
Generalized Fibonacci Search Method in One-Dimensional Unconstrained Non-Linear Optimization
}

\author{
Chin Yoon Chong ${ }^{*}$, Soo Kar Leow ${ }^{1}$ and Hong Seng Sim ${ }^{2}$ \\ ${ }^{1}$ School of Mathematics Sciences, Sunway University, Jalan Universiti, Bandar Sunway, 47500 Selangor, Malaysia \\ ${ }^{2}$ Department of Mathematical and Actuarial Sciences, Universiti Tunku Abdul Rahman, \\ 43000 Kajang, Selangor, Malaysia
}

\begin{abstract}
In this paper, we develop a generalized Fibonacci search method for one-dimensional unconstrained non-linear optimization of unimodal functions. This method uses the idea of the "ratio length of 1" from the golden section search. Our method takes successive lower Fibonacci numbers as the initial ratio and does not specify beforehand, the number of iterations to be used. We evaluated the method using Microsoft Excel with nine onedimensional benchmark functions. We found that our generalized Fibonacci search method out-performed the golden section and other Fibonacci-type search methods such as the Fibonacci, Lucas and Pell approaches.
\end{abstract}

Keywords: Fibonacci-type, Generalized Fibonacci search, golden section

\section{INTRODUCTION}

Many techniques have been introduced to solve the problem of the one-dimensional unconstrained non-linear optimization, i.e. to minimize (or maximize) a unimodal function $f(x)$, where $x$ is real.

ARTICLE INFO

Article history:

Received: 18 October 2020

Accepted: 14 January 2021

Published: 30 April 2021

DOI: https://doi.org/10.47836/pjst.29.2.17

E-mail addresses:

cychong@sunway.edu.my (Chin Yoon Chong)

skleow@sunway.edu.my (Soo Kar Leow)

simhs@utar.edu.my (Hong Seng Sim)

* Corresponding author
In this paper, we will focus more on the Fibonacci-type search methods. We first study the importance of the bracketing search method (Bazaraa et al., 2013; Belegundu \& Chandrupatla, 2019; Hassin, 1981; Rao, 2019). Subsequently, we proceed to the golden section search (Beamer \& Wilde, 1969; Luenberger \& Ye, 1984; Solomon, 2015; Rao, 2019), which is a technique to achieve the optimal of a unimodal function 
within a specified interval by involving a ratio, $r=\frac{1}{2}(\sqrt{5}-1)$ such that $r=\varphi^{-1}$, where $\varphi=\frac{1}{2}(\sqrt{5}+1)$, namely, the golden ratio (Bazaraa et al., 2013; Belegundu \& Chandrupatla, 2019; Solomon, 2015). Next, we further our research to the well-known Fibonacci search method (Witzgall, 1969; Kiefer, 1953; Avriel \& Wilde, 1966; Hassin, 1981).

By applying the idea of "ratio length of 1", we will introduce a different approach to obtain the ratio $(r)$ of the golden section search in one-dimensional optimization problems, and we also propose a different approach of Fibonacci search by putting the successive lower Fibonacci numbers as the initial ratios. Next, by introducing the sub-Fibonacci numbers, we will extend our results to a new search method, called the generalized Fibonacci search. This is the main emphasis in our research. Like the golden section search, both the Fibonacci search and generalized Fibonacci search methods have similar algorithms where their ratios are changed at every iteration.

Based on the necessary and sufficient conditions obtained in the generalized Fibonacci search, together with the bracketing search upon the initial interval with same width, we use Microsoft Excel to test nine one-dimensional benchmark functions and compare our results with the golden section search and other Fibonacci-type search methods such as the Fibonacci, Lucas and Pell (Koshy, 2019). Furthermore, the Fibonacci-like numbers of Mersenne, Jacobsthal (Catarino et al., 2016), arithmetic or geometric (Chong \& Ho, 2015) will also be discussed.

\section{Fibonacci-Like Numbers}

A famous Italian mathematician, Leonardo Fibonacci (Pisa, c.1170 - Pisa, c.1240) wrote on a variety of mathematical topics and Fibonacci is remembered particularly for the sequence numbers as $0,1,1,2,3,5,8,13,21,34, \ldots$, to which his name has been applied. This sequence, even today, is the subject of continuing research, especially by the Fibonacci Association, which publishes The Fibonacci Quarterly. The Fibonacci numbers are recursively defined by $F_{n+2}=F_{n+1}+F_{n}$ for all integers $n \geq 0$, with initial values $F_{0}=0, F_{1}=1$ (Koshy, 2019).

Marin Mersenne (Maine, 8 September 1588 - Paris, 1 September 1648), a French mathematician, is perhaps best known today among mathematicians for the Mersenne prime numbers, those which can be written in the form $M_{n}=2^{n}-1$. This can be described as a sequence of Fibonacci-like numbers namely Mersenne numbers as $M_{0}=0, M_{1}=1$, $M_{n+2}=3 M_{n+1}-2 M_{n}$ (Catarino et al., 2016).

In seventeenth-century, an English Mathematician, John Pell (Southwick, 1 March 1611 Westminster, 12 December 1685) defined a sequence of numbers $0,1,2,5,12,29, \ldots$, also known as Pell numbers, which can be recursively generated as $P_{0}=0, P_{1}=0, P_{n+2}=2 P_{n+1}+P_{n}$. Pell numbers can be used to find square triangular numbers, to construct integer approximations 
to the right isosceles triangle, and to solve certain combinatorial enumeration problems (Catarino et al., 2016; Kilic, 2007).

Jackques Philippe Marrie Binet (Rennes, 2 February 1786 - Paris, 12 May 1856), a French mathematician developed a Binet formula in 1843 that directly calculate the value of the $n$th Fibonacci number, that is, $F_{n}=\frac{1}{\sqrt{5}}\left[\left(\frac{1+\sqrt{5}}{2}\right)^{n}-\left(\frac{1-\sqrt{5}}{2}\right)^{n}\right]$, where $\frac{1 \pm \sqrt{5}}{2}$ are the roots of $x^{2}+x+1=0$. The well-known golden ratio also can be obtained from the limiting value on the ratio of two successive Fibonacci numbers as $n$ approaches infinity, i.e., $\varphi=\lim _{n \rightarrow \infty} \frac{F_{n}}{F_{n-1}}=\frac{\sqrt{5}+1}{2}$. However, Binet was not the first to find this expression. De Moivre had discovered it much earlier in 1718 (Koshy, 2019).

Another French mathematician, Édouard Anatole Lucas (Amiens, 4 April 1842 - Paris, 3 October 1891) succeeded in branching out to another type of sequence, that is: 2, 1, 3, 4, $7,11,18,29,47, \ldots$, which is named after him as the Lucas numbers. The Lucas number is denoted recursively as $L_{n+2}=L_{n+1}+L_{n}$, with the initial values $L_{0}=2$ and $L_{1}=1$. The Lucas numbers are closely related to the Fibonacci numbers (Koshy, 2019).

In 1965, Alwyn Francis Horadam (Hunter Valley, 22 March 1923 - Armidale, July 2016), an Australian mathematician introduced a Horadom-generalized Fibonacci number $H_{n}$, which is defined by $H_{n+2}=p H_{n+1}+q H_{n}$ with initial values $H_{0}=a, H_{1}=b$ , where $a, b, p$ and $q$ are real constants (Horadam, 1965; Horzum \& Kocer, 2009; Udrea, 1996). He developed and obtained the generalized Fibonacci numbers by giving a very useful generalized Binet formula as follows, $H_{n}=\frac{1}{\alpha-\beta}\left[(b-a \beta) \alpha^{n}-(b-a \alpha) \beta^{n}\right]$, where $\alpha=\frac{p+\sqrt{p^{2}+4 q}}{2}$ and $\beta=\frac{p-\sqrt{p^{2}+4 q}}{2}$ are two distinct real roots of the quadratic equation $x^{2}-p x-q=0 . \alpha$ is known as the generalized golden ratio which is also the limit of the ratio of two successive generalized Fibonacci numbers when $n$ approaches infinity, that is, $\varphi=\lim _{n \rightarrow \infty} \frac{H_{n}}{H_{n-1}}=\frac{p+\sqrt{p^{2}+4 q}}{2}$.

\section{Golden Section Search and Fibonacci Search}

An American mathematical statistician, Jack Carl Kiefer (Cincinnati, 25 January 1924 - Berkeley, 10 August 1981) discovered the golden section search and the well-known Fibonacci search (Kiefer, 1953). These are the sequential methods for seeking the optimum of a unimodal function $f(x)$ as the golden section search needs a fixed ratio $\varphi^{-1}$, where $\varphi$ is the golden ratio, while the ratio of the Fibonacci search varies in every stage of the iterations. 
The theoretical advantage of the zero-order methods, like the Fibonacci and the golden section search. Thus, these search methods are widely popular as their algorithms do not require differentiability of the unimodal function $f(x)$ as an underlying assumption. In practice, the golden section search is frequently used when the total number of iterations required need not be specified.

Unlike the golden section procedure, the Fibonacci method requires the total number of iterations be chosen beforehand. For $n$ large enough, then $\frac{F_{n-1}}{F_{n}}=\varphi^{-1}$, and thus the Fibonacci search method and the golden section are identical. It is worth mentioning that among the derivative-free methods that minimize strict unimodal functions over a closed bounded interval, the Fibonacci search method is the most efficient in that it requires the smallest number of iterations for a given reduction in the length of the interval of uncertainty (Bazaraa et al., 2013; Belegundu \& Chandrupatla, 2019; Hassin, 1981; Luenberger \& Ye, 1984; Solomon, 2015).

Subasi et al. (2004) searched the optimal point by Lucas numbers in the Fibonacci search algorithm, the improved method gave better results, where the optimal values of the objective functions were more sensitive in the improved method when their results were examined by a developed computer program in MAPLE. Demir et al. (2008) presented a mathematical analysis of the Fibonacci search method by $k$-Lucas numbers. In this study, they developed a new algorithm which determined the maximum point of unimodal functions on closed intervals. As a result, it made the Fibonacci search method more effective.

\section{MATERIALS AND METHODS}

In this section, we will focus on the line search algorithm for the one-dimensional optimization problem. We will describe the bracketing, the golden section, the Fibonacci and the generalized Fibonacci search. Further, we will also discuss the conditions to be fulfilled for the generalized Fibonacci search. We will close this section with the comparison of our results with the results from the golden section search and the other Fibonacci-type search methods.

\section{Unimodal Function}

A unimodal function is one that has only one peak (maximum) or valley (minimum) in a given domain. For instance, a univariate function $f(x)$ is unimodal on an interval $\left[x_{1}, x_{2}\right]$ if there exists a minimum $x^{*} \in\left(x_{1}, x_{2}\right)$ such that $f(x)$ is monotonically decreasing for $x \in\left[x_{1}, x^{*}\right]$ and monotonically increasing for $x \in\left[x^{*}, x_{2}\right]$. In other words, there are no other local minima on the interval $\left(x_{1}, x_{2}\right)$ (Avriel \& Wilde, 1966; Beamer \& Wilde, 1969; Hassin, 1981; Kiefer, 1953; Oliver \& Wilde, 1964; Witzgall, 1969). 
It is perhaps more conventional to define unimodal functions in the opposite sense, such that there is a unique global maximum rather than a minimum. However, since any maximization problem can be transformed into a minimization problem by multiplying the function by a negative sign. Moreover, a unimodal function with an optimum $x^{*} \in\left(x_{1}, x_{2}\right)$ can be a nondifferentiable or even a discontinuous function.

For the rest of the paper, we will assume $f(x)$ is a unimodal function which has a minimizer $x^{*}$ in the interval $\left(x_{1}, x_{2}\right)$, that is $x_{1}<x^{*}<x_{2}$. Let $x_{3}$ and $x_{4}$ be the two points such that $x_{1}<x_{3}<x_{4}<x_{2}$.

\section{Bracketing Search Method}

The search for a local optimum $x *$ will involve a sequence of function evaluations for some different points of $x \in\left[x_{1}, x_{2}\right]$ One after the other, using the previous results of the function evaluations to obtain the new interval that bounds the optimum $x^{*}$. At each iteration, the closer interval that brackets the optimum $x^{*}$ is called the interval of uncertainty. From the past, bracketing search has been discussed by many researchers (Avriel \& Wilde, 1966; Belegundu \& Chandrupatla, 2019; Hassin, 1981; Oliver \& Wilde, 1964, Solomon, 2015; Witzgall, 1969). We have two possibilities of the interval of uncertainty as follows.

\section{Case 1}

If $f\left(x_{3}\right)<f\left(x_{4}\right)$, then $x_{1}<x^{*}<x_{3}<x_{4}<x_{2}$ or $x_{1}<x_{3}<x^{*}<x_{4}<x_{2}$. Thus, $x^{*} \in\left(x_{1}^{\prime}, x_{2}^{\prime}\right)$ such that $x_{1}^{\prime}=x_{1}$ and $x_{2}^{\prime}=x_{4}$.

\section{Case 2}

If $f\left(x_{3}\right)>f\left(x_{4}\right)$, then $x_{1}<x_{3}<x_{4}<x^{*}<x_{2}$ or $x_{1}<x_{3}<x^{*}<x_{4}<x_{2}$. Thus, $x^{*} \in\left(x_{1}^{\prime}, x_{2}^{\prime}\right)$ such that $x_{1}^{\prime}=x_{3}$ and $x_{2}^{\prime}=x_{2}$.

\section{Remark}

$\left|x_{2}^{\prime}-x_{1}^{\prime}\right|<\left|x_{2}-x_{1}\right|$ for the above two cases.

\section{Golden Section Search Method}

The ratio $r$ of the golden section search plays a very important role in line search method as described above. This ratio, $r$ acts as a "generator" to generate the values of $x_{3}$ and $x_{4}$ from the initial interval $\left[x_{1}, x_{2}\right]$. The following algorithm shows the procedure how can the ratio $r$ be obtained.

Let $x_{2}-x_{1}=d$ and $x_{3}-x_{1}=x_{2}-x_{4}$ in the first iteration as shown in Figure 1. 
Let $r$ be a ratio such that $\frac{x_{4}-x_{1}}{x_{2}-x_{1}}=\frac{x_{2}-x_{3}}{x_{2}-x_{1}}=r$, where $0<r<1$.

Then $x_{4}-x_{1}=x_{2}-x_{3}=r d$ gives $x_{3}-x_{1}=x_{2}-x_{4}=(1-r) d$

Simultaneously, let $\frac{x_{3}-x_{1}}{x_{4}-x_{1}}=\frac{x_{2}-x_{4}}{x_{2}-x_{3}}=r$. Then $x_{3}-x_{1}=x_{2}-x_{4}=r^{2} d$

Equation 1 and 2 will give $r=\frac{1}{2}(\sqrt{5}-1)$. Moreover, by substituting $d=x_{2}-x_{1}$ into Equation 1 yields $x_{4}=(1-r) x_{1}+r x_{2}$ and $x_{4}=(1-r) x_{1}+r x_{2}$, respectively. Together with the bracketing search, the process will obtain an interval of uncertainty for which $x^{*} \in\left(x_{1}^{\prime}, x_{2}^{\prime}\right)$. Without loss of generality, at $m$ th iteration, then

$$
x_{3}^{(m-1)}=r x_{1}^{(m-1)}+(1-r) x_{2}^{(m-1)} \text { and } x_{4}^{(m-1)}=(1-r) x_{1}^{(m-1)}+r x_{2}^{(m-1)}
$$

The similar algorithm has been discussed in some articles (Bazaraa et al., 2013; Solomon, 2015; Belegundu \& Chandrupatla, 2019).

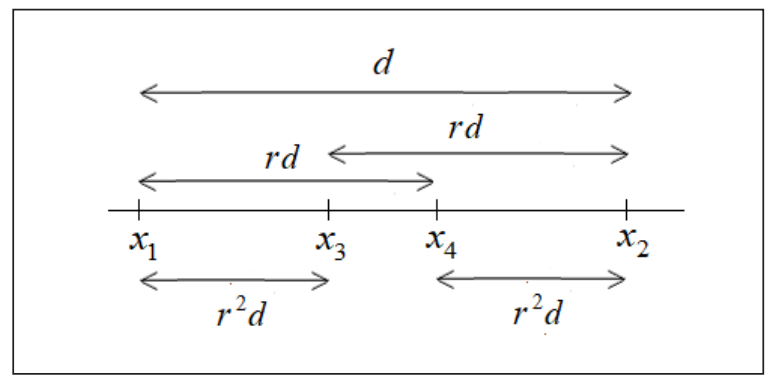

Figure 1. Length measured in terms of $r$ and $d$

\section{Different Approach to Obtain the Ratio ( $r$ ) of Golden Section Search}

In this section, we introduce a different approach to obtain the ratio $r$ of the golden section search by applying the idea of "ratio length of 1 ".

Let $x_{2}-x_{1}=1$ and $x_{3}-x_{1}=x_{2}-x_{4}$, as shown in Figure 2. In other words, we treat the length, $x_{2}-x_{1}$ as a total ratio of 1 , and based on the same algorithm as discussed above, we will obtain the same results, where the length from the diagram is measured in terms of $r$.

This idea of "ratio length of 1" will lead us to a different approach of the Fibonacci search, and also provide us an idea to develop a new search method, that is the generalized Fibonacci search. This will be discussed in the next section of Different Approach of Fibonacci Search, and Generalized Fibonacci Search as well. 


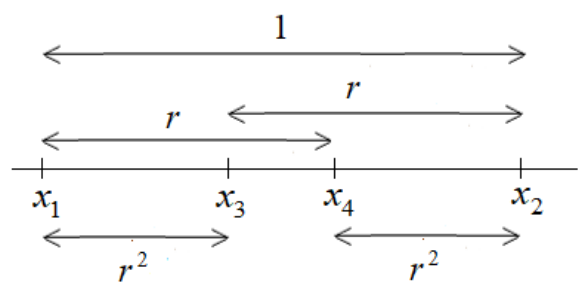

Figure 2. Length measured in terms of $r$

\section{Fibonacci Search with a Fixed Number of Iterations}

In this section, we first describe the Fibonacci search as mentioned by Bazaraa et al. (2013), Belegundu and Chandrupatla (2019), Hassin (1981), Mathews et al. (2004), Subasi et al. (2004), which starts with a ratio with two successive higher Fibonacci numbers, that is $\frac{F_{n-2}}{F_{n}}$ for $m$ iterations.

${ }^{F_{n}}$ Golden section search and Fibonacci search have a very close relationship, and thus we first to investigate the initial ratio of the Fibonacci search by comparing the ratio of the golden section search. Suppose $x_{2}-x_{1}=d$ and $x_{3}-x_{1}=x_{2}-x_{4}$, as illustrated in Figure 3. Assume a fixed number of iterations, $m$ is needed to achieve the desired optimal $x^{*}$.

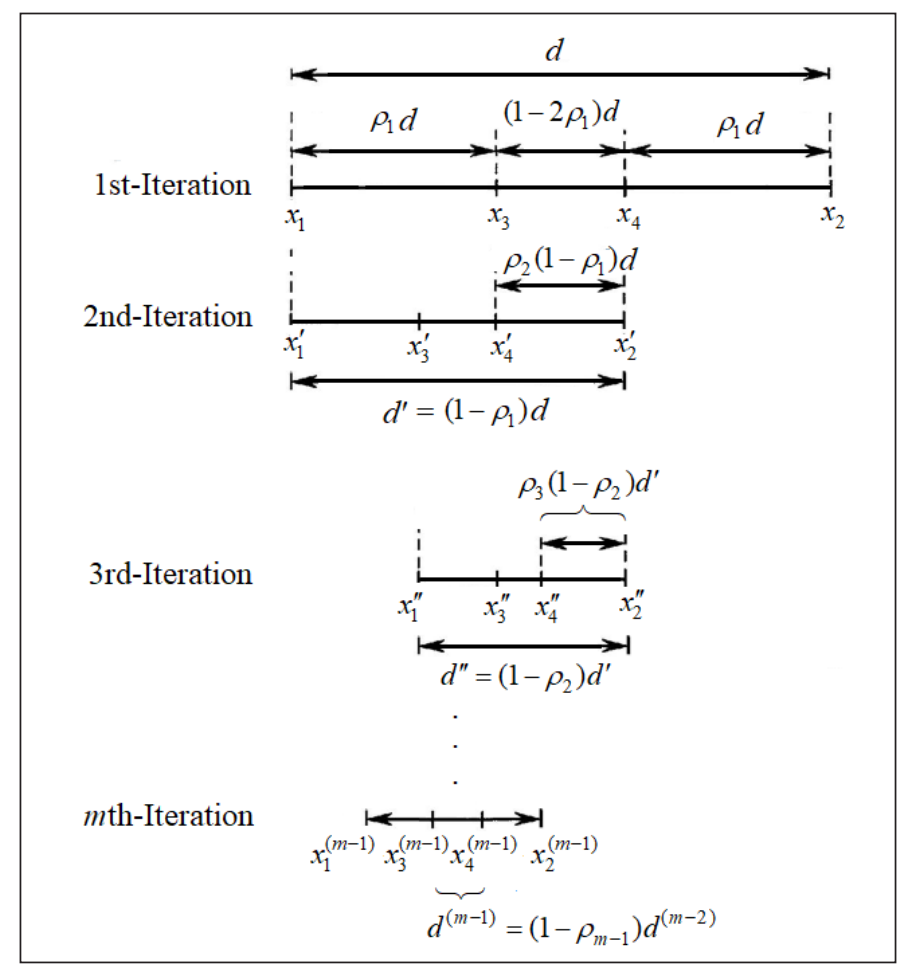

Figure 3. Initial ratio that has higher Fibonacci numbers 
In the first iteration, we let $\frac{x_{3}-x_{1}}{x_{2}-x_{1}}=\frac{x_{2}-x_{4}}{x_{2}-x_{1}}=\rho_{1}$, where $0<\rho_{1}<1$, then $x_{4}-x_{1}=\left(1-\rho_{1}\right) d$. Compared to $x_{4}-x_{1}=r d$ in Figure 1, therefore we have $1-\rho_{1}=r$. Since all the search methods will give a high efficiency when $n \rightarrow \infty$, therefore, $1-\rho_{1}=r=\frac{1}{\phi}=\lim _{n \rightarrow \infty} \frac{1}{\frac{F_{n}}{F_{n-1}}}=\lim _{n \rightarrow \infty} \frac{F_{n-1}}{F_{n}}$ gives $\rho_{1}=1-\frac{F_{n-1}}{F_{n}}=\frac{F_{n-2}}{F_{n}}$ as $n$ approaches infinity. Hence, this is proven that the initial ratio of the Fibonacci search will involve the successive higher Fibonacci numbers. Next, we will obtain a new interval of uncertainty, where $x^{*} \in\left(x_{1}^{\prime}, x_{2}^{\prime}\right)$ after the bracketing search is imposed.

In the second iteration, let $d^{\prime}=x_{2}^{\prime}-x_{1}^{\prime}=x_{4}-x_{1}=\left(1-\rho_{1}\right) d$ such that $x_{2}^{\prime}-x_{4}^{\prime}=x_{4}-x_{3}$ and exists a ratio $\rho_{2}$ for which $\frac{x_{3}^{\prime}-x_{1}^{\prime}}{x_{2}^{\prime}-x_{1}^{\prime}}=\frac{x_{2}^{\prime}-x_{4}^{\prime}}{x_{2}^{\prime}-x_{1}^{\prime}}=\rho_{2}$. Hence, $\rho_{2}\left(1-\rho_{1}\right) d=\left(1-2 \rho_{1}\right) d$ gives $\rho_{2}=\frac{1-2 \rho_{1}}{1-\rho_{1}}=\frac{1-\frac{2 F_{n-2}}{F_{n}}}{1-\frac{F_{n-2}}{F_{n}}}=\frac{F_{n}-2 F_{n-2}}{F_{n}-F_{n-2}}=\frac{F_{n-3}}{F_{n-1}}$.

Next, the bracketing search gives a new interval of uncertainty such that $d^{\prime \prime}=\left(1-\rho_{2}\right) d^{\prime}$. Repeat the above process, then $d^{\prime \prime}=\left(1-\rho_{2}\right) d^{\prime}$ and $\rho_{3}=\frac{F_{n-4}}{F_{n-2}}$ in the third iteration.

Generally, $d^{(m-1)}=\left(1-\rho_{m-1}\right) d^{(m-2)}$ and $\rho_{m}=\frac{F_{n-m-1}}{F_{n-m+1}}$ in $m$ th iteration.

Thus, when $m=n-2$, the results obtained:

$$
\begin{aligned}
& d^{(n-3)}=\left(1-\rho_{n-3}\right) d^{(n-4)}=\left(1-\rho_{n-3}\right)\left(1-\rho_{n-4}\right) \cdots\left(1-\rho_{3}\right)\left(1-\rho_{2}\right)\left(1-\rho_{1}\right) d \\
& =\left(1-\frac{F_{2}}{F_{4}}\right)\left(1-\frac{F_{3}}{F_{5}}\right) \cdots\left(1-\frac{F_{n-4}}{F_{n-2}}\right)\left(1-\frac{F_{n-3}}{F_{n-1}}\right)\left(1-\frac{F_{n-2}}{F_{n}}\right) d \\
& =\frac{F_{3}}{F_{4}} \frac{F_{4}}{F_{5}} \cdots \frac{F_{n-2}}{F_{n-1}} \frac{F_{n-1}}{F_{n}} d \\
& =\frac{F_{3} d}{F_{n}}
\end{aligned}
$$

\section{Fibonacci Search Without a Fixed Number of Iterations}

What if we begin the ratio with the two successive lower Fibonacci numbers and without a fixed number of iterations? We will propose an algorithm procedure that begins with the 
first ratio with lower Fibonacci numbers and the results will show that both procedures are equivalent. This result brews an idea and lead us to develop the new approach of search method in next sections.

Let $x_{2}-x_{1}=d$ and $x_{3}-x_{1}=x_{2}-x_{4}$, as shown in Figure 4. Next, we proposed another method by considering the assumption of first ratio with the lower Fibonacci numbers as $\lambda_{1}=\frac{F_{2}}{F_{4}}$ in the first iteration such that $\frac{x_{3}-x_{1}}{x_{2}-x_{1}}=\frac{x_{2}-x_{4}}{x_{2}-x_{1}}=\lambda_{1}$, and thus $x_{4}-x_{3}=\left(1-2 \lambda_{1}\right) d$. Subsequently, we will obtain a new interval of uncertainty for which $x^{*} \in\left(x_{1}^{\prime}, x_{2}^{\prime}\right)$ upon the bracketing search.

In the second iteration with the assumption by taking another ratio with onestep ahead of two successive lower Fibonacci numbers as $\lambda_{2}=\frac{F_{3}}{F_{5}}$, and let $D^{\prime}=x_{2}^{\prime}-x_{1}^{\prime}=x_{4}-x_{1}=\left(1-\lambda_{1}\right) d$ such that $\frac{x_{3}^{\prime}-x_{1}^{\prime}}{x_{2}^{\prime}-x_{1}^{\prime}}=\frac{x_{2}^{\prime}-x_{4}^{\prime}}{x_{2}^{\prime}-x_{1}^{\prime}}=\lambda_{2}$. Take note that $x_{2}^{\prime}-x_{4}^{\prime}$ and $x_{4}-x_{3}$ are not necessarily in equal length, whereby $x_{2}^{\prime}-x_{4}^{\prime}=x_{4}-x_{3}$ in Figure 3. Next, the bracketing search gives a new interval of uncertainty such that $x^{*} \in\left(x_{1}^{\prime \prime}, x_{2}^{\prime \prime}\right)$.

As such, without loss of generality, we will have $D^{\prime \prime}=\left(1-\lambda_{2}\right) D^{\prime}$ and $\lambda_{3}=\frac{F_{4}}{F_{6}}$ in the third iteration, and $x^{*} \in\left(x_{1}^{\prime \prime}, x_{2}^{\prime \prime}\right)$. Generally, $D^{(m-1)}=\left(1-\lambda_{m-1}\right) D^{(m-2)}$ and $\lambda_{m}=\frac{F_{m+1}}{F_{m+3}}$ in $m$ th iteration, where $D^{(0)}=d$. Thus, when $m=n-2$, we get

$$
\begin{aligned}
& D^{(n-3)}=\left(1-\lambda_{n-3}\right) D^{(n-4)}=\left(1-\lambda_{n-3}\right)\left(1-\lambda_{n-4}\right) \cdots\left(1-\lambda_{3}\right)\left(1-\lambda_{2}\right)\left(1-\lambda_{1}\right) d \\
& =\left(1-\frac{F_{n-2}}{F_{n}}\right)\left(1-\frac{F_{n-3}}{F_{n-1}}\right) \cdots\left(1-\frac{F_{3}}{F_{5}}\right)\left(1-\frac{F_{2}}{F_{4}}\right) d \\
& =\frac{F_{n-1}}{F_{n}} \frac{F_{n-2}}{F_{n-1}} \cdots \frac{F_{4}}{F_{5}} \frac{F_{3}}{F_{4}} d \\
& =\frac{F_{3} d}{F_{n}}
\end{aligned}
$$

which is equal to the result of $d^{(n-3)}$ in Figure 3. This is proven that the Fibonacci search can be iterated with the first ratio of successive lower Fibonacci numbers. Hence, compared to the previous method, this will advantage us to run the algorithm with the successive lower Fibonacci numbers and without a fixed number of iterations at the beginning. In other words, we could see the limitation in fixing $m$ iterations at the start of the algorithm. 


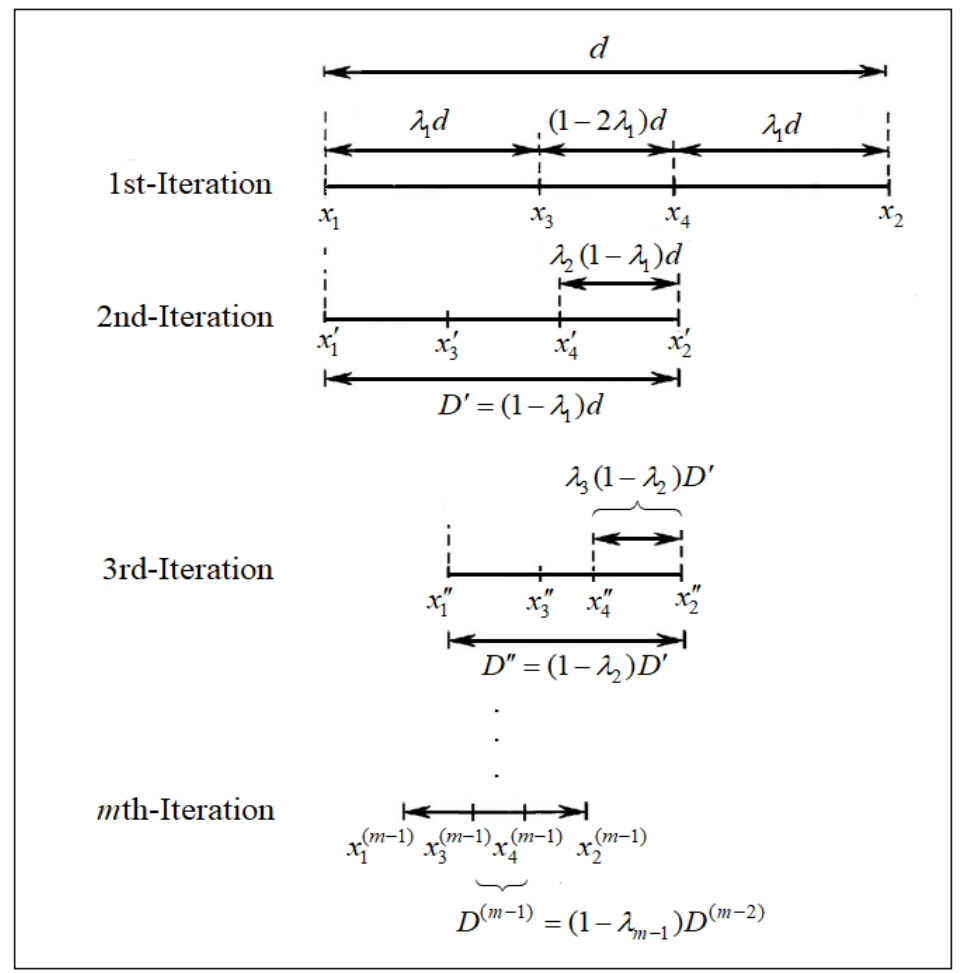

Figure 4. Initial ratio that has lower Fibonacci numbers

\section{Different Approach of Fibonacci Search}

In the following section, we proposed a different approach of the Fibonacci search method compared to the Fibonacci search as discussed by Bazaraa et al. (2013), Belegundu and Chandrupatla (2019), Hassin (1981), and Subasi et al. (2004).

Let $F_{n}$ be the Fibonacci number which is defined recursively by $F_{0}=0, F_{1}=1$ and $F_{n+2}=F_{n+1}+F_{n}$ for all integers $n \geq 0$. Based on the idea of "ratio length of 1 " as discussed in the golden section search above, we will consider the successive lower Fibonacci numbers, i.e., $\frac{F_{2}}{F_{4}}$ and $\frac{F_{3}}{F_{4}}$ as the initial ratios in the first iteration. From Figure 5, we have Equation 3

$$
\frac{x_{2}-x_{3}}{x_{2}-x_{1}}=\frac{x_{4}-x_{1}}{x_{2}-x_{1}}=\frac{F_{3}}{F_{4}} \text { and } \frac{x_{3}-x_{1}}{x_{2}-x_{1}}=\frac{x_{2}-x_{4}}{x_{2}-x_{1}}=\frac{F_{2}}{F_{4}} \text {. }
$$

Then the following equalities hold:

$$
\frac{x_{2}-x_{3}}{x_{2}-x_{1}}+\frac{x_{3}-x_{1}}{x_{2}-x_{1}}=\frac{F_{3}}{F_{4}}+\frac{F_{2}}{F_{4}}=1 \text { and } \frac{x_{4}-x_{1}}{x_{2}-x_{1}}+\frac{x_{2}-x_{4}}{x_{2}-x_{1}}=\frac{F_{3}}{F_{4}}+\frac{F_{2}}{F_{4}}=1 .
$$


Hence, from Equation 3, we obtained the following results, $x_{3}=\frac{F_{3}}{F_{4}} x_{1}+\frac{F_{2}}{F_{4}} x_{2}$ and $x_{4}=\frac{F_{2}}{F_{4}} x_{1}+\frac{F_{3}}{F_{4}} x_{2}$.

Using the bracketing search, we will find the new interval of uncertainty for $x^{*} \in\left(x_{1}^{\prime}, x_{2}^{\prime}\right)$, where $\left|x_{2}^{\prime}-x_{1}^{\prime}\right|<\left|x_{2}-x_{1}\right|$.

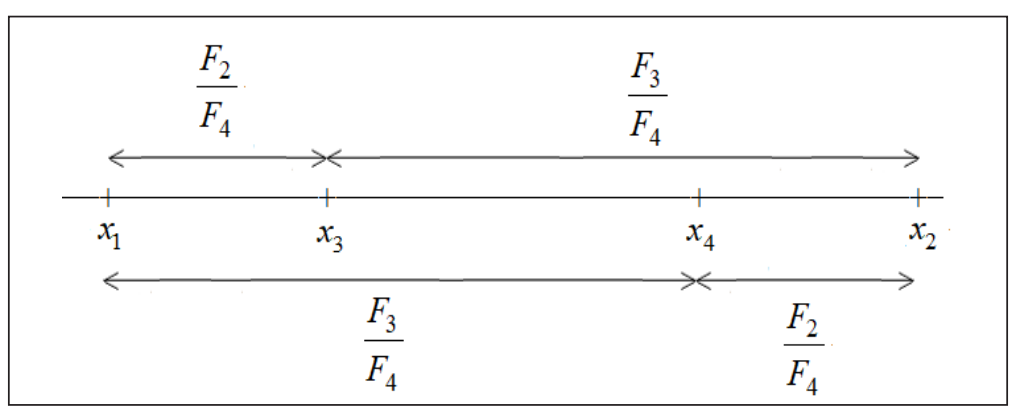

Figure 5. Ratios with lower Fibonacci numbers at 1st iteration

Subsequently, with the same process at the second iteration, we consider different ratios of one-step ahead of two successive Fibonacci numbers $\frac{F_{3}}{F_{5}}$ and $\frac{F_{4}}{F_{5}}$, and we let $x_{3}^{\prime}$ and $x_{4}^{\prime}$ be two points for which $x_{1}^{\prime}<x_{3}^{\prime}<x_{4}^{\prime}<x_{2}^{\prime}$, such that

$$
\frac{x_{2}^{\prime}-x_{3}^{\prime}}{x_{2}^{\prime}-x_{1}^{\prime}}=\frac{x_{4}^{\prime}-x_{1}^{\prime}}{x_{2}^{\prime}-x_{1}^{\prime}}=\frac{F_{4}}{F_{5}} \text { and } \frac{x_{3}^{\prime}-x_{1}^{\prime}}{x_{2}^{\prime}-x_{1}^{\prime}}=\frac{x_{2}^{\prime}-x_{4}^{\prime}}{x_{2}^{\prime}-x_{1}^{\prime}}=\frac{F_{3}}{F_{5}}
$$

subject to $\frac{x_{2}^{\prime}-x_{3}^{\prime}}{x_{2}^{\prime}-x_{1}^{\prime}}+\frac{x_{3}^{\prime}-x_{1}^{\prime}}{x_{2}^{\prime}-x_{1}^{\prime}}=\frac{F_{4}}{F_{5}}+\frac{F_{3}}{F_{5}}=1$ and $\frac{x_{4}^{\prime}-x_{1}^{\prime}}{x_{2}^{\prime}-x_{1}^{\prime}}+\frac{x_{2}^{\prime}-x_{4}^{\prime}}{x_{2}^{\prime}-x_{1}^{\prime}}=\frac{F_{4}}{F_{5}}+\frac{F_{3}}{F_{5}}=1$.

Then, Equation 4, we will obtain the following results, $x_{3}^{\prime}=\frac{F_{4}}{F_{5}} x_{1}^{\prime}+\frac{F_{3}}{F_{5}} x_{2}^{\prime}$ and $x_{4}^{\prime}=\frac{F_{3}}{F_{5}} x_{1}^{\prime}+\frac{F_{4}}{F_{5}} x_{2}^{\prime}$.

Next, we will obtain a new interval of uncertainty for $x^{*} \in\left(x_{1}^{\prime \prime}, x_{2}^{\prime \prime}\right)$ after the bracketing search is imposed, where $\left|x_{2}^{\prime \prime}-x_{1}^{\prime \prime}\right|<\left|x_{2}^{\prime}-x_{1}^{\prime}\right|$.

Repeat the above process until $m$ th iteration as shown in Figure 6 so that

$$
\frac{x_{2}^{(m-1)}-x_{3}^{(m-1)}}{x_{2}^{(m-1)}-x_{1}^{(m-1)}}=\frac{x_{4}^{(m-1)}-x_{1}^{(m-1)}}{x_{2}^{(m-1)}-x_{1}^{(m-1)}}=\frac{F_{m+2}}{F_{m+3}} \text { and } \frac{x_{3}^{(m-1)}-x_{1}^{(m-1)}}{x_{2}^{(m-1)}-x_{1}^{(m-1)}}=\frac{x_{2}^{(m-1)}-x_{4}^{(m-1)}}{x_{2}^{(m-1)}-x_{1}^{(m-1)}}=\frac{F_{m+1}}{F_{m+3}}
$$


subject to $\frac{x_{2}^{(m-1)}-x_{3}^{(m-1)}}{x_{2}^{(m-1)}-x_{1}^{(m-1)}}+\frac{x_{3}^{(m-1)}-x_{1}^{(m-1)}}{x_{2}^{(m-1)}-x_{1}^{(m-1)}}=\frac{F_{m+2}}{F_{m+3}}+\frac{F_{m+1}}{F_{m+3}}=1$

and $\frac{x_{4}^{(m-1)}-x_{1}^{(m-1)}}{x_{2}^{(m-1)}-x_{1}^{(m-1)}}+\frac{x_{2}^{(m-1)}-x_{4}^{(m-1)}}{x_{2}^{(m-1)}-x_{1}^{(m-1)}}=\frac{F_{m+2}}{F_{m+3}}+\frac{F_{m+1}}{F_{m+3}}=1$.

Thus, Equation 5 gives the following results,

$$
x_{3}^{(m-1)}=\frac{F_{m+2}}{F_{m+3}} x_{1}^{(m-1)}+\frac{F_{m+1}}{F_{m+3}} x_{2}^{(m-1)} \text { and } x_{4}^{(m-1)}=\frac{F_{m+1}}{F_{m+3}} x_{1}^{(m-1)}+\frac{F_{m+2}}{F_{m+3}} x_{2}^{(m-1)}
$$

such that $x_{1}^{(m-1)}<x_{3}^{(m-1)}<x_{4}^{(m-1)}<x_{2}^{(m-1)}$, where $\frac{F_{m+1}}{F_{m+3}}<\frac{F_{m+2}}{F_{m+3}}$ for all $m \geq 1$.

Next, the bracketing search yields a closer interval of uncertainty for which $x^{*} \in\left(x_{1}^{m)}, x_{2}^{(m)}\right)$ such that $\left|x_{2}^{(m)}-x_{1}^{(m)}\right|<\left|x_{2}^{(m-1)}-x_{1}^{(m-1)}\right|$, where $\left|x_{2}^{(m)}-x_{1}^{(m)}\right| \approx 0$ as $m \rightarrow \infty$.

The proof of the results of $x_{3}^{(m-1)}$ and $x_{4}^{(m-1)}$ is as follows:

At $m$ th iteration, since $\frac{x_{2}^{(m-1)}-x_{3}^{(m-1)}}{x_{2}^{(m-1)}-x_{1}^{(m-1)}}=\frac{F_{m+2}}{F_{m+3}}$, therefore $x_{2}^{(m-1)}-x_{3}^{(m-1)}=\frac{F_{m+2}}{F_{m+3}}\left[x_{2}^{(m-1)}-x_{1}^{(m-1)}\right]$ gives $x_{3}^{(m-1)}=\frac{F_{m+2}}{F_{m+3}} x_{1}^{(m-1)}+\left(1-\frac{F_{m+2}}{F_{m+3}}\right) x_{2}^{(m-1)}=\frac{F_{m+2}}{F_{m+3}} x_{1}^{(m-1)}+\frac{F_{m+1}}{F_{m+3}} x_{2}^{(m-1)}$.

Similarly, $\frac{x_{4}^{(m-1)}-x_{1}^{(m-1)}}{x_{2}^{(m-1)}-x_{1}^{(m-1)}}=\frac{F_{m+2}}{F_{m+3}}$ gives $x_{4}^{(m-1)}=\frac{F_{m+1}}{F_{m+3}} x_{1}^{(m-1)}+\frac{F_{m+2}}{F_{m+3}} x_{2}^{(m-1)}$.

Alternatively, $\frac{x_{3}^{(m-1)}-x_{1}^{(m-1)}}{x_{2}^{(m-1)}-x_{1}^{(m-1)}}=\frac{x_{2}^{(m-1)}-x_{4}^{(m-1)}}{x_{2}^{(m-1)}-x_{1}^{(m-1)}}=\frac{F_{m+1}}{F_{m+3}}$ also gives the same results of $x_{4}^{(m-1)}$ and $x_{4}^{(m-1)}$.

Take note that the above results of $x_{3}^{(m-1)}$ and $x_{4}^{(m-1)}$ matched the results of golden section search as $m$ approaches infinity. Since $\lim _{m \rightarrow \infty} \frac{F_{m+2}}{F_{m+3}}=\lim _{m \rightarrow \infty} \frac{1}{\frac{F_{m+3}}{F_{m+2}}}=\frac{1}{\varphi}=r$, therefore the above results will give $x_{3}^{(m-1)}=\frac{F_{m+2}}{F_{m+3}} x_{1}^{(m-1)}+\frac{F_{m+1}}{F_{m+3}} x_{2}^{(m-1)}=\frac{F_{m+2}}{F_{m+3}} x_{1}^{(m-1)}+\left(1-\frac{F_{m+2}}{F_{m+3}}\right) x_{2}^{(m-1)}$

$$
=r x_{1}^{(m-1)}+(1-r) x_{2}^{(m-1)}
$$

as $m \rightarrow \infty$. Thus, similarly for $x_{4}^{(m-1)}$, which will equal to $(1-r) x_{1}^{(m-1)}+r x_{2}^{(m-1)}$. 


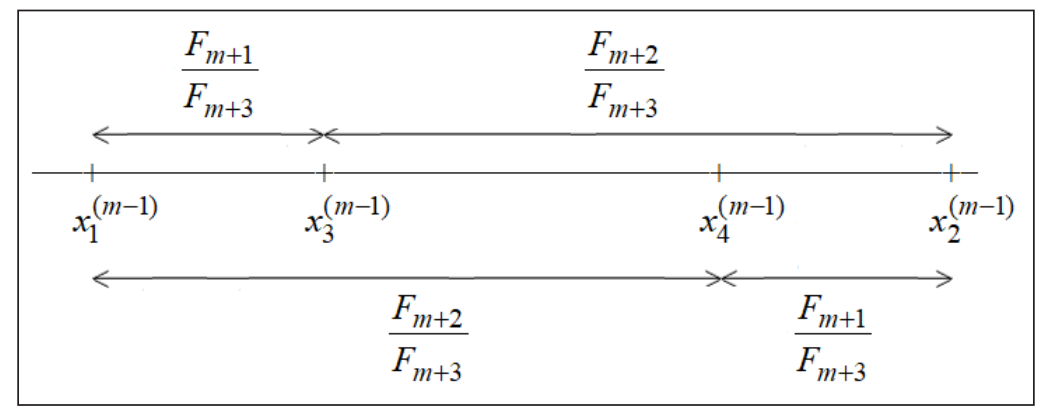

Figure 6. Ratios with higher Fibonacci numbers at $m$ th iteration

\section{Generalized Fibonacci Search}

The generalized Fibonacci search method is the main focus of our research. It may have more complexities compared to the Fibonacci search. Unlike the Fibonacci search discussed previously, not all the Fibonacci-like numbers can be applied in the algorithm of the generalized Fibonacci search method. Thus, to satisfy the algorithm, we have to restrict the conditions of the combination of $a, b, p$ and $q$ from the generalized Fibonacci number (Horadam, 1965), $H_{n}=p H_{n-1}+q H_{n-2}, H_{0}=a, H_{1}=b$ for all real values of $a$, $b, p$ and $q$. Thus, we will introduce a new term, sub-Fibonacci number before proceeding to discussion of the generalized Fibonacci search.

Definition 1. Let $a$ and $b$ be two nonnegative integers such that $a+b>0$. Let $p$ and $q$ be two positive integers. Then $S_{n}(a, b, p, q)$ denote the $n$th sub-Fibonacci number for all integers $n \geq 0$, where $S_{0}=a, S_{1}=b$ and $S_{n+2}=p S_{n+1}+q S_{n}$.

Lemma 1. If $S_{n}(a, b, p, q)$ is an $n$th sub-Fibonacci number, then $S_{n}>0$ for all $n \geq 2$.

Proof. Since $S_{n}(a, b, p, q)$ gives $S_{0}=a \geq 0, S_{1}=b \geq 0$ and $p, q>0$, where $a$ and $b$ never take zero simultaneously, therefore $S_{n}=p S_{n-1}+q S_{n-2}>0$ for all integers $n \geq 2$. We complete the proof.

\section{Algorithm of Generalized Fibonacci Search}

Let $S_{n}(a, b, p, q)$ be the $n$th sub-Fibonacci number for all integers $n \geq 0$. We begin the algorithm with the initial ratios of the successive lower Fibonacci numbers for $n \geq 2$, i.e., we use $\frac{q S_{2}}{S_{4}}$ and $\frac{p S_{3}}{S_{4}}$ in the first iteration as shown in Figure 7, we have Equation 6

$$
\frac{x_{3}-x_{1}}{x_{2}-x_{1}}=\frac{x_{2}-x_{4}}{x_{2}-x_{1}}=\frac{q S_{2}}{S_{4}} \text { and } \frac{x_{3}-x_{1}}{x_{2}-x_{1}}=\frac{x_{2}-x_{4}}{x_{2}-x_{1}}=\frac{q S_{2}}{S_{4}}
$$


subject to $\frac{x_{2}-x_{3}}{x_{2}-x_{1}}+\frac{x_{3}-x_{1}}{x_{2}-x_{1}}=\frac{p S_{3}}{S_{4}}+\frac{q S_{2}}{S_{4}}=1$ and $\frac{x_{4}-x_{1}}{x_{2}-x_{1}}+\frac{x_{2}-x_{4}}{x_{2}-x_{1}}=\frac{p S_{3}}{S_{4}}+\frac{q S_{2}}{S_{4}}=1$.

From Equation 6, we obtained the following results, $x_{3}=\frac{p S_{3}}{S_{4}} x_{1}+\frac{q S_{2}}{S_{4}} x_{2}$ and $x_{4}=\frac{q S_{2}}{S_{4}} x_{1}+\frac{p S_{3}}{S_{4}} x_{2}$.

We proceed to the bracketing search to find the new interval of uncertainty such that $x^{*} \in\left(x_{1}^{\prime}, x_{2}^{\prime}\right)$, where $\left|x_{2}^{\prime}-x_{1}^{\prime}\right|<\left|x_{2}-x_{1}\right|$.

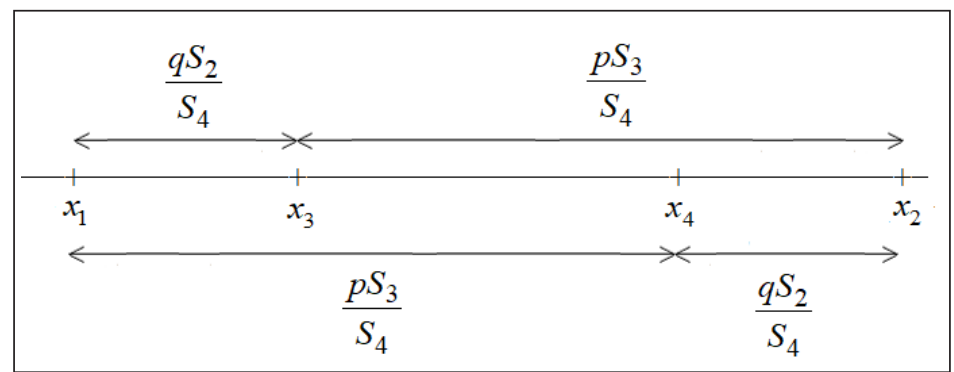

Figure 7. Ratios with lower generalized Fibonacci numbers at 1 st iteration

Next, we repeat the above process for the second iteration with different ratios of onestep ahead of two successive sub-Fibonacci numbers, i.e., $\frac{q S_{3}}{S_{5}}$ and $\frac{p S_{4}}{S_{5}}$. Let $x_{3}^{\prime}$ and $x_{4}^{\prime}$ be another two points for which $x_{1}^{\prime}<x_{3}^{\prime}<x_{4}^{\prime}<x_{2}^{\prime}$, such that:

$$
\frac{x_{2}^{\prime}-x_{3}^{\prime}}{x_{2}^{\prime}-x_{1}^{\prime}}=\frac{x_{4}^{\prime}-x_{1}^{\prime}}{x_{2}^{\prime}-x_{1}^{\prime}}=\frac{p S_{4}}{S_{5}} \text { and } \frac{x_{3}^{\prime}-x_{1}^{\prime}}{x_{2}^{\prime}-x_{1}^{\prime}}=\frac{x_{2}^{\prime}-x_{4}^{\prime}}{x_{2}^{\prime}-x_{1}^{\prime}}=\frac{q S_{3}}{S_{5}}
$$

subject to $\frac{x_{2}^{\prime}-x_{3}^{\prime}}{x_{2}^{\prime}-x_{1}^{\prime}}+\frac{x_{3}^{\prime}-x_{1}^{\prime}}{x_{2}^{\prime}-x_{1}^{\prime}}=\frac{p S_{4}}{S_{5}}+\frac{q S_{3}}{S_{5}}=1$ and $\frac{x_{4}^{\prime}-x_{1}^{\prime}}{x_{2}^{\prime}-x_{1}^{\prime}}+\frac{x_{2}^{\prime}-x_{4}^{\prime}}{x_{2}^{\prime}-x_{1}^{\prime}}=\frac{p S_{4}}{S_{5}}+\frac{q S_{3}}{S_{5}}=1$.

Then, from Equation 7, we will obtain the following results,

$$
x_{3}^{\prime}=\frac{p S_{4}}{S_{5}} x_{1}^{\prime}+\frac{q S_{3}}{S_{5}} x_{2}^{\prime} \text { and } x_{4}^{\prime}=\frac{q S_{3}}{S_{5}} x_{1}^{\prime}+\frac{p S_{4}}{S_{5}} x_{2}^{\prime} .
$$

Continuing with the bracketing search, yields another new interval of uncertainty for $x^{*} \in\left(x_{1}^{\prime \prime}, x_{2}^{\prime \prime}\right)$, where $\left|x_{2}^{\prime \prime}-x_{1}^{\prime \prime}\right|<\left|x_{2}^{\prime}-x_{1}^{\prime}\right|$. Subsequently, after the third and fourth iteration, we will have the respective results: 


$$
\begin{aligned}
& x_{3}^{\prime \prime}=\frac{p S_{5}}{S_{6}} x_{1}^{\prime \prime}+\frac{q S_{4}}{S_{6}} x_{2}^{\prime \prime}, x_{4}^{\prime \prime}=\frac{q S_{4}}{S_{6}} x_{1}^{\prime \prime}+\frac{p S_{5}}{S_{6}} x_{2}^{\prime \prime}, \text { where } x_{1}^{\prime \prime}<x_{3}^{\prime \prime}<x_{4}^{\prime \prime}<x_{2}^{\prime \prime} \text { and give } x^{*} \in\left(x_{1}^{\prime \prime \prime}, x_{2}^{\prime \prime \prime}\right), \\
& x_{3}^{\prime \prime \prime}=\frac{p S_{6}}{S_{7}} x_{1}^{\prime \prime \prime}+\frac{q S_{5}}{S_{7}} x_{2}^{\prime \prime \prime}, \quad x_{4}^{\prime \prime \prime}=\frac{q S_{5}}{S_{7}} x_{1}^{\prime \prime \prime}+\frac{p S_{6}}{S_{7}} x_{2}^{\prime \prime \prime}, \quad \text { wher e } x_{1}^{\prime \prime \prime}<x_{3}^{\prime \prime \prime}<x_{4}^{\prime \prime \prime}<x_{2}^{\prime \prime \prime} \text { and g i v e } \\
& x^{*} \in\left(x_{1}^{(4)}, x_{2}^{(4)}\right) .
\end{aligned}
$$

Thus, generally at $m$ th iteration, suppose $x_{3}^{(m-1)}$ and $x_{4}^{(m-1)}$ are the two new points after the bracketing search is imposed, where $x_{1}^{(m-1)}<x_{3}^{(m-1)}<x_{4}^{(m-1)}<x_{2}^{(m-1)}$, as shown in Figure 8 , such that

$$
\frac{x_{2}^{(m-1)}-x_{3}^{(m-1)}}{x_{2}^{(m-1)}-x_{1}^{(m-1)}}=\frac{x_{4}^{(m-1)}-x_{1}^{(m-1)}}{x_{2}^{(m-1)}-x_{1}^{(m-1)}}=\frac{p S_{m+2}}{S_{m+3}} \text { and } \frac{x_{3}^{(m-1)}-x_{1}^{(m-1)}}{x_{2}^{(m-1)}-x_{1}^{(m-1)}}=\frac{x_{2}^{(m-1)}-x_{4}^{(m-1)}}{x_{2}^{(m-1)}-x_{1}^{(m-1)}}=\frac{q S_{m+1}}{S_{m+3}}[8]
$$

s u b j e c t t o $\frac{x_{2}^{(m-1)}-x_{3}^{(m-1)}}{x_{2}^{(m-1)}-x_{1}^{(m-1)}}+\frac{x_{3}^{(m-1)}-x_{1}^{(m-1)}}{x_{2}^{(m-1)}-x_{1}^{(m-1)}}=\frac{p S_{m+2}}{S_{m+3}}+\frac{q S_{m+1}}{S_{m+3}}=1 \quad$ a $\mathrm{n} \mathrm{d}$ $\frac{x_{4}^{(m-1)}-x_{1}^{(m-1)}}{x_{2}^{(m-1)}-x_{1}^{(m-1)}}+\frac{x_{2}^{(m-1)}-x_{4}^{(m-1)}}{x_{2}^{(m-1)}-x_{1}^{(m-1)}}=\frac{p S_{m+2}}{S_{m+3}}+\frac{q S_{m+1}}{S_{m+3}}=1$.

Then, Equation 8 gives the results of $x_{3}^{(m-1)}$ and $x_{4}^{(m-1)}$ as

$$
x_{3}^{(m-1)}=\frac{p S_{m+2}}{S_{m+3}} x_{1}^{(m-1)}+\frac{q S_{m+1}}{S_{m+3}} x_{2}^{(m-1)} \text { and } x_{4}^{(m-1)}=\frac{q S_{m+1}}{S_{m+3}} x_{1}^{(m-1)}+\frac{p S_{m+2}}{S_{m+3}} x_{2}^{(m-1)} .
$$

Next, the bracketing search yields the closer interval of uncertainty for $x^{*} \in\left(x_{1}^{(m)}, x_{2}^{(m)}\right)$ such that $\left|x_{2}^{(m)}-x_{1}^{(m)}\right|<\mid x_{2}^{(m-1)}-x_{1}^{(m-1)}$, where $\left|x_{2}^{(m)}-x_{1}^{(m)}\right| \approx 0$ as $m \rightarrow \infty$.

The following is the proof for the results of $x_{3}^{(m-1)}$ and $x_{4}^{(m-1)}$.

At $m$ thiteration, since $\frac{x_{2}^{(m-1)}-x_{3}^{(m-1)}}{x_{2}^{(m-1)}-x_{1}^{(m-1)}}=\frac{p S_{m+2}}{S_{m+3}}$, then $x_{2}^{(m-1)}-x_{3}^{(m-1)}=\frac{p S_{m+2}}{S_{m+3}}\left[x_{2}^{(m-1)}-x_{1}^{(m-1)}\right]$ gives $x_{3}^{(m-1)}=\frac{p S_{m+2}}{S_{m+3}} x_{1}^{(m-1)}+\left(1-\frac{p S_{m+2}}{S_{m+3}}\right) x_{2}^{(m-1)}=\frac{p S_{m+2}}{S_{m+3}} x_{1}^{(m-1)}+\frac{q S_{m+1}}{S_{m+3}} x_{2}^{(m-1)}$.

Similarly, $\frac{x_{4}^{(m-1)}-x_{1}^{(m-1)}}{x_{2}^{(m-1)}-x_{1}^{(m-1)}}=\frac{p S_{m+2}}{S_{m+3}}$ gives $x_{4}^{(m-1)}=\frac{q S_{m+1}}{S_{m+3}} x_{1}^{(m-1)}+\frac{p S_{m+2}}{S_{m+3}} x_{2}^{(m-1)}$. 
Alternatively, $\frac{x_{3}^{(m-1)}-x_{1}^{(m-1)}}{x_{2}^{(m-1)}-x_{1}^{(m-1)}}=\frac{x_{2}^{(m-1)}-x_{4}^{(m-1)}}{x_{2}^{(m-1)}-x_{1}^{(m-1)}}=\frac{q S_{m+1}}{S_{m+3}}$ also give the same results $x_{3}^{(m-1)}$ and $x_{4}^{(m-1)}$.

\begin{tabular}{|c|c|c|c|}
\hline & \multicolumn{3}{|c|}{$\underline{p S_{m+2}}$} \\
\hline$x_{1}^{(m-1)}$ & $x_{3}^{(m-1)}$ & $x_{4}^{(m-1)}$ & $x_{2}^{(m-1)}$ \\
\hline & $\frac{p S_{m+2}}{S_{m+3}}$ & & \\
\hline
\end{tabular}

Figure 8. Ratios with higher generalized Fibonacci numbers at $m$ th iteration

Not all the sub-Fibonacci numbers will fulfill the algorithm with different sets of combinations of $a, b, p$ and $q$. Next, we will present the necessary and sufficient conditions of $a, b, p$ and $q$ to ensure the convergence of the optimal solution as follows.

Lemma 2. Consider an $n$th sub-Fibonacci number, $S_{n}(a, b, p, q)$ for all integers $n=k \geq 0$. If $q S_{k}<p S_{k+1}$ and $q S_{k+1}<p S_{k+2}$, then $q S_{i}<p S_{i+1}$ for all $i \geq k+2$.

Proof. We shall prove this by mathematical induction for $S_{n}(a, b, p, q)$, where $a+b>0$ and $n=k \geq 0$. Suppose the results of the following Equation 9 and 10 are true for $n=k \geq 0$. i.e.,

$$
q S_{k}<p S_{k+1} \text { gives } \frac{q^{2}}{p} S_{k}<q S_{k+1}
$$

and $q S_{k+1}<p S_{k+2}$

Now $q S_{k+2}=q\left(p S_{k+1}+q S_{k}\right)=p\left(q S_{k+1}+\frac{q^{2}}{p} S_{k}\right)<p\left(p S_{k+2}+q S_{k+1}\right)=p S_{k+3}$

Thus, Equation 11 show that the result is also true for $n=k+1$. Similarly, Equation 10 and 11 will lead to $q S_{k+3}<p S_{k+4}$. We complete the proof.

Definition 2. An optimal $x^{*}$ of a unimodal function $f(x)$ is achievable if and only if $x^{*}$ can be approximated by any line search method such that $x *$ is bounded within all the intervals of uncertainty. 
Theorem 1. Let $S_{n}(a, b, p, q)$ denote the $n$th sub-Fibonacci number for all for all $n \geq 0$ . Then the following statements are equivalent such that an optimal $x *$ of a unimodal function $f(x)$ is achievable by the generalized Fibonacci search for all $n \geq 2$ if and only if

(a) $q S_{n}<p S_{n+1}$.

(b) $0<\frac{q S_{n}}{S_{n+2}}<\frac{1}{2}<\frac{p S_{n+1}}{S_{n+2}}<1$.

(c) $q\left(q-p^{2}\right) a<p^{3} b$ and $q\left(q-p^{2}\right) b<p^{3}(q a+p b)$.

Proof. Consider $S_{n}(a, b, p, q)>0$ for all $n \geq 2$ such that $a+b>0$ and $p, q>0$.

(a) For all $m \geq 1$, the generalized Fibonacci search at $m$ th iteration gives $x_{3}^{(m-1)}=\frac{p S_{m+2}}{S_{m+3}} x_{1}^{(m-1)}+\frac{q S_{m+1}}{S_{m+3}} x_{2}^{(m-1)} \quad$ and $x_{4}^{(m-1)}=\frac{q S_{m+1}}{S_{m+3}} x_{1}^{(m-1)}+\frac{p S_{m+2}}{S_{m+3}} x_{2}^{(m-1)}$.

So, $x^{*}$ is achievable if and only if $x_{1}^{(m-1)}<x_{3}^{(m-1)}<x_{4}^{(m-1)}<x_{2}^{(m-1)}$.

Thus, $x_{3}^{(m-1)}<x_{4}^{(m-1)}$ gives $\frac{p S_{m+2}}{S_{m+3}} x_{1}^{(m-1)}+\frac{q S_{m+1}}{S_{m+3}} x_{2}^{(m-1)}<\frac{q S_{m+1}}{S_{m+3}} x_{1}^{(m-1)}+\frac{p S_{m+2}}{S_{m+3}} x_{2}^{(m-1)}$

$$
\begin{aligned}
& \frac{q S_{m+1}}{S_{m+3}}\left(x_{2}^{(m-1)}-x_{1}^{(m-1)}\right)<\frac{p S_{m+2}}{S_{m+3}}\left(x_{2}^{(m-1)}-x_{1}^{(m-1)}\right) \\
& q S_{m+1}<p S_{m+2}
\end{aligned}
$$

Obviously, $q S_{n}<p S_{n+1}$ for all $n \geq 2$. Thus, we complete the proof.

(b) From (a), $q S_{n}<p S_{n+1}$ gives $0<\frac{q S_{n}}{S_{n+2}}<\frac{p S_{n+1}}{S_{n+2}}$. Now, since $\frac{p S_{n+1}}{S_{n+2}}+\frac{q S_{n}}{S_{n+2}}=1$, therefore $\frac{q S_{n}}{S_{n+2}}<\frac{1}{2}$ and $\frac{p S_{n+1}}{S_{n+2}}>\frac{1}{2}$. Also, $S_{n+2}=p S_{n+1}+q S_{n}$ gives $S_{n+2}>p S_{n+1}$, i.e., $\frac{p S_{n+1}}{S_{n+2}}<1$. Hence, the proof is completed.

(c) Since $q S_{2}<p S_{3}$ gives $q\left(q-p^{2}\right) a<p^{3} b \quad$ and $\quad q S_{3}<p S_{4} \quad$ gives $q\left(q-p^{2}\right) b<p^{3}(q a+p b)$, thus, Lemma 2 gives $q S_{i}<p S_{i+1}$ for all $i \geq 4$. Next, Theorem 1(a) completes the proof. 
Theorem 2. Let $S_{n}(a, b, p, q)$ denote the $n$th sub-Fibonacci number for all $n \geq 0$. An optimal $x^{*}$ of a unimodal function $f(x)$ is achievable by generalized Fibonacci search for all $n \geq 2$ if either one of the following is true.

(a) $q<p^{2}$

(b) $q=p^{2}$ and $b>0$.

Proof. Consider an $n$th sub-Fibonacci number, $S_{n}(a, b, p, q)$ such that $a+b>0$ and $p, q>0$.

(a) Notice that $S_{0}=a \geq 0$ and $S_{1}=b \geq 0$, where $a$ and $b$ never take zero simultaneously. If $q<p^{2}$, then

$$
\begin{aligned}
& q S_{1}-p S_{2}=q S_{1}-p\left(p S_{1}+q S_{0}\right)=\left(q-p^{2}\right) b-p q a<0 \\
& \text { And } q S_{2}-p S_{3}=q S_{2}-p\left(p S_{2}+q S_{1}\right)=q\left(q-p^{2}\right) a-p^{3} b<0
\end{aligned}
$$

Lemma 2 leads Equation 12 and 13 to generate $q S_{i}<p S_{i+1}$ for all $i \geq 3$. Next, Theorem 1(a) completes the proof.

(b) Since $p^{2}=q, a \geq 0$ and $b>0$, therefore

$$
q S_{2}-p S_{3}=q S_{2}-p\left(p S_{2}+q S_{1}\right)=q\left(q-p^{2}\right) a-p^{3} b=-p^{3} b<0
$$

and

$$
q S_{3}-p S_{4}=q S_{3}-p\left(p S_{3}+q S_{2}\right)=\left[q\left(q-p^{2}\right)-p^{4}\right] b-p^{3} q a=-p^{3}(q a+p b)<0
$$

Lemma 2 leads Equation 14 and 15 to produce $q S_{i}<p S_{i+1}$ for all $i \geq 4$. Then, Theorem 1 (a) completes the proof.

\section{RESULTS AND DISCUSSIONS}

Table 1 shows the benchmark functions of two variables obtained from Test Functions for Optimization at the following link: https:/en.wikipedia.org/wiki/Test_functions_for_ optimization.

We converted the two-dimensional benchmark functions to a one-dimensional function by fixing the values of $y$ in $f(x, y)$ with the optimal $y$-coordinate, $y^{*}$, or vice-versa the values of $x$ in $f(x, y)$ with the optimal $x^{*}$. Then we searched the cross section along the 
lines $y=y^{*}$ or $x=x^{*}$ using the line search method for one variable since $f\left(x, y^{*}\right)$ and $f\left(x^{*}, y\right)$ have only one degree of freedom.

We implemented the generalized Fibonacci search with $a=2, b=7, p=2$ and $q=$ 7 , namely Fibo2727, which is involved the sub-Fibonacci numbers, $S_{n}(2,7,2,7)$. Based on the same initial intervals of $\left[x_{1}, x_{2}\right]$ and compared to the golden section search and other Fibonacci-type search methods such as Fibonacci, Lucas, or Pell, we found that experimentally tested with a self-developed function in Microsoft Excel, Fibo2727 could give the least number of iterations to achieve the optimization. Pell is the weakest, as shown in Table 2, where the optimal value has rounded up to 7 decimal places.

Figure 9 shows the flow chart of Fibonacci-like numbers. Not all the Fibonacci-like numbers will fulfil the generalized Fibonacci search method. For instance, the Mersenne

Table 1

Benchmark functions of two variables

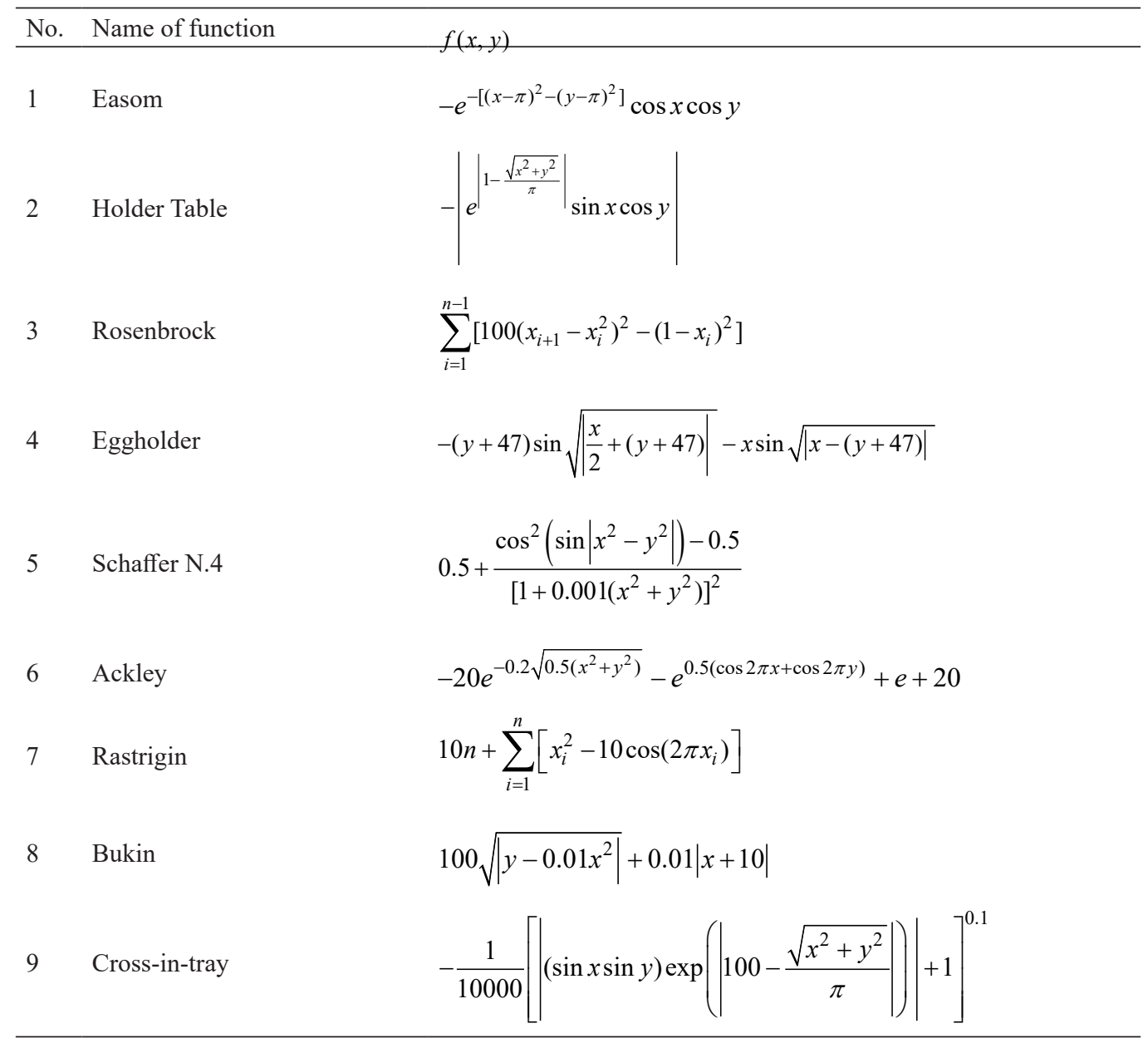


Table 2

Comparison of the performance of golden section search and other Fibonacci-type search methods on benchmark functions

\begin{tabular}{|c|c|c|c|c|c|c|c|c|}
\hline \multirow{3}{*}{ No. } & \multirow{3}{*}{ Function } & \multirow{3}{*}{$\begin{array}{c}\text { Initial } \\
\text { interval } \\
\left(x_{1}, x_{2}\right)\end{array}$} & \multirow{3}{*}{$\begin{array}{l}\text { Optimal } \\
\text { (min) }\end{array}$} & \multicolumn{5}{|c|}{ Fibonacci-Type $(a, b, p, q)$} \\
\hline & & & & $\begin{array}{l}\text { Golden } \\
\text { Section }\end{array}$ & $\begin{array}{l}\text { Fibonacci } \\
(0,1,1,1)\end{array}$ & $\begin{array}{c}\text { Lucas } \\
(2,1,1,1)\end{array}$ & $\begin{array}{c}\text { Pell } \\
(0,1,2,1)\end{array}$ & $\begin{array}{r}\text { Fibo2727 } \\
(2,7,2,7) \\
\end{array}$ \\
\hline & & & & \multicolumn{5}{|c|}{ Number of Iterations } \\
\hline 1 & Easom at $\mathrm{y}=\pi$ & $(2,4)$ & $\begin{array}{c}x *= \\
3.1415927\end{array}$ & 35 & 35 & 35 & 88 & 26 \\
\hline 2 & $\begin{array}{l}\text { Holder Table at } \\
y=9.66459\end{array}$ & $(6,9)$ & $\begin{array}{c}x^{*}= \\
8.0550236\end{array}$ & 35 & 37 & 36 & 90 & 26 \\
\hline 3 & $\begin{array}{l}\text { Rosenbrock at } y \\
=1, n=2\end{array}$ & $(0,3)$ & $x^{*}=1$ & 38 & 38 & 36 & 93 & 28 \\
\hline 4 & $\begin{array}{l}\text { Eggholder at } x \\
=512\end{array}$ & $\begin{array}{l}(350, \\
450)\end{array}$ & $\begin{array}{c}x^{*}= \\
404.2318503\end{array}$ & 40 & 41 & 41 & 105 & 30 \\
\hline 5 & $\begin{array}{l}\text { Schaffer N.4 at } \\
x=0\end{array}$ & $(0,3)$ & $\begin{array}{c}x *= \\
1.2531318\end{array}$ & 38 & 38 & 37 & 90 & 28 \\
\hline 6 & Ackley at $x=0$ & $(-2,1.5)$ & $y^{*}=0$ & 38 & 38 & 38 & 83 & 28 \\
\hline 7 & $\begin{array}{l}\text { Rastrigin at } x= \\
0, n=2\end{array}$ & $(-1,3)$ & $y^{*}=0$ & 39 & 38 & 39 & 97 & 29 \\
\hline 8 & Bukin at $y=1$ & $(-20,0)$ & $x^{*}=-10$ & 42 & 42 & 42 & 104 & 31 \\
\hline 9 & $\begin{array}{l}\text { Cross-in-tray at } \\
y=1.3494064\end{array}$ & $(0,2)$ & $\begin{array}{c}x^{*}= \\
1.3494064\end{array}$ & 34 & 36 & 34 & 87 & 24 \\
\hline
\end{tabular}

(Catarino et al., 2016), arithmetic or geometric numbers (Chong \& Ho, 2015) failed in generalized Fibonacci search method since they were not the members of sub-Fibonacci number as their $q$ 's values were negative. However, although the Jacobsthal number is a sub-Fibonacci number (Catarino et al., 2016), but it also failed as well as it does not fulfil Theorem 1.

The results significantly show that the algorithm of both the Fibonacci search and generalized Fibonacci search methods can be expressed with the initial ratio of two successive lower Fibonacci numbers in the first iteration. This will lead us to investigate the combination of the values of $a, b, p, q$ to further improve our search algorithm in future. We will also attempt to extend the generalized Fibonacci search method to two-dimensional or higher dimensional optimization problems of unimodal functions.

\section{CONCLUSIONS}

In this paper, we found another approach to obtain the ratio of the golden section search, which led us to an idea of the "ratio length of 1". This idea opened up a big step for us to explore deeper research in Fibonacci search, because $F_{n+2}=F_{n+1}+F_{n}$ always give 


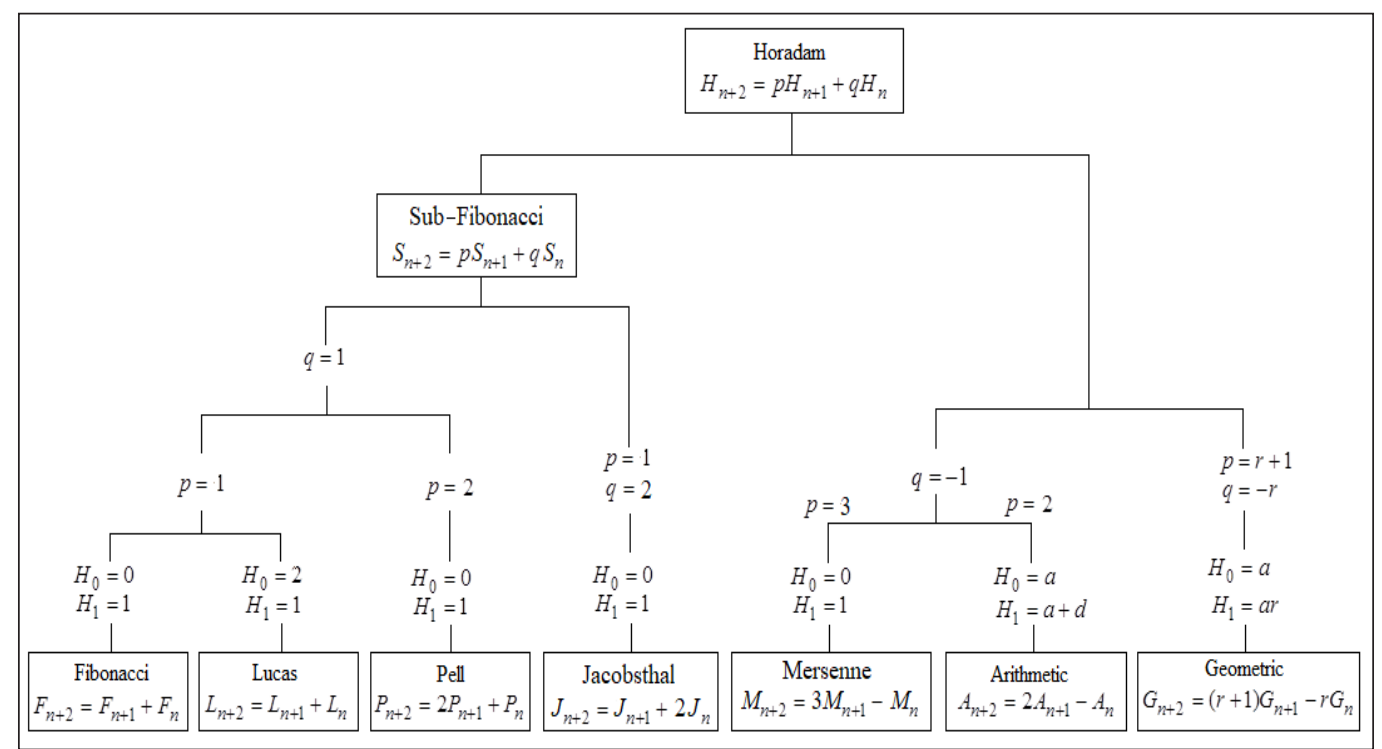

Figure 9. Flow chart of Fibonacci-like numbers

$\frac{F_{n+1}}{F_{n+2}}+\frac{F_{n}}{F_{n+2}}=1$. Additionally, we proved that both the algorithms of the Fibonacci and the generalized Fibonacci search could be iterated with the successive lower Fibonacci numbers. As such, from the generalized Fibonacci number $S_{4}=p S_{3}+q S_{2}$, where $\frac{p S_{3}}{S_{4}}+\frac{q S_{2}}{S_{4}}=1$, we considered $\frac{q S_{2}}{S_{4}}$ and $\frac{p S_{3}}{S_{4}}$ as the initial ratios for the first iteration. Hence this would advantage us to run the algorithm without fixing the number of iterations.

Unfortunately, certain sub-Fibonacci numbers failed the algorithm of the generalized Fibonacci search. However, we found the necessary and sufficient conditions of $a, b, p$ and $q$ to ensure the convergence of the optimal solution.

In the last section of this paper, we compared the performance of the generalized Fibonacci search with the golden section and other line Fibonacci-type search methods such as Fibonacci, Lucas and Pell approaches using nine one-dimensional benchmark functions. Based on the same width of the initial interval for each search method, we used a self-developed function in Microsoft Excel to test a number of different combination values of $a, b, p$ and $q$, the generalized Fibonacci search performed more effectively and successfully gave significant results, correct to 7 decimal places, when $a=2, b=7, p=$ 2 and $q=7$. In other words, the generalized Fibonacci search with $S_{n}(2,7,2,7)$ required less iterations to achieve the optimal when compared to others.

We strongly believe that there is no best combination of $a, b, p$ and $q$ to achieve the optimum by the generalized Fibonacci search. We shall attempt to prove this statement in future. 


\section{ACKNOWLEDGEMENT}

The authors wish to express their gratitude to the School of Mathematical Sciences, Sunway University for the financial support in the conduct of this research.

\section{REFERENCES}

Avriel, M., \& Wilde, D. J. (1966). Optimally proof for the symmetric Fibonacci search technique. Fibonacci Quarterly Journal, 265-269.

Bazaraa, M. S., Sherali, H. D., \& Shetty, C. M. (2013). Nonlinear programming: Theory and algorithms. John Wiley \& Sons.

Beamer, J. H., \& Wilde, D. J. (1971). Minimax optimization of a unimodal function by variable block derivative search with time delay. Journal of Combinatorial Theory, Series A, 10(2), 160-173. https:// doi.org/10.1016/0097-3165(71)90019-7

Belegundu, A. D., \& Chandrupatla, T. R. (2019). Optimization concepts and applications in engineering. Cambridge University Press.

Catarino, P., Campos, H., \& Vasco, P. (2016). On the Mersenne sequence. CM-Centro de Matemática, 46, 37-53.

Chong, C. Y., \& Ho, C. K. (2015, December). Some Properties of generalized Fibonacci sequence. In AIP Conference Proceedings (Vol. 1691, No. 1, p. 040004). AIP Publishing LLC. https://doi. org/10.1063/1.4937054

Demir, A., Omur, N., \& Ulutas, Y. T. (2008). Optimization by k-Lucas numbers. Applied mathematics and computation, 197(1), 366-371. https://doi.org/10.1016/j.amc.2007.07.045

Hassin, R. (1981). On maximizing functions by Fibonacci search. Fibonacci Quarterly, 19, 347-351.

Horadam, A. F. (1965). Basic properties of a certain generalized sequence of numbers. The Fibonacci Quarterly, 3(3), 161-176.

Horzum, T., \& Kocer, E. G. (2009). On some properties of Horadam polynomials. International Mathematical Forum, 4(25), 1243-1252.

Kiefer, J. (1953). Sequential minimax search for a maximum. Proceedings of the American Mathematical Society, 4(3), 502-506. https://doi.org/10.2307/2032161

Kilic, E. (2007). The generalized order-k Fibonacci-Pell sequence by matrix methods. Journal of Computational and Applied Mathematics, 209(2), 133-145. https://doi.org/10.1016/j.cam.2006.10.071

Koshy, T. (2019). Fibonacci and Lucas numbers with applications (2nd Ed.). John Wiley \& Sons.

Luenberger, D. G., \& Ye, Y. (1984). Linear and nonlinear programming (Vol. 2). Addison-wesley.

Mathews, J. H., \& Fink, K. D. (2004). Numerical methods using MATLAB (Vol. 4). Pearson prentice hall.

Oliver, L. T., \& Wilde, D. J. (1964). Symmetric sequential minimax search for a maximum. Fibonacci Quarterly, 3, 169-176.

Rao, S. S. (2019). Engineering optimization: Theory and practice. John Wiley \& Sons. 
Solomon, J. (2015). Numerical algorithms: Methods for computer vision, machine learning, and graphics. CRC press.

Subasi, M., Yildirim, N., \& Yildiz, B. (2004). An improvement on Fibonacci search method in optimization theory. Applied mathematics and computation, 147(3), 893-901. https://doi.org/10.1016/S00963003(02)00828-7

Udrea, G. (1996). A note on the sequence $\left(W_{n}\right)_{n \geq 0}$ of AF Horadam. Portugaliae Mathematica, 53(2), 143-156.

Witzgall, C. (1969). Fibonacci search with arbitrary first evaluation. Boeing Scientific Research Labs Seattle Wa Mathematics Research Lab. 
